\title{
String and particle with two times
}

\author{
Itzhak Bars* and Costas Kounnas ${ }^{\dagger}$ \\ Theory Division, CERN, CH-1211 Geneva 23, Switzerland
}

(Received 27 May 1997)

\begin{abstract}
An action for a string and a particle with two timelike dimensions is proposed and analyzed. Because of the new gauge symmetries and associated constraints, the motion of each system in the background of the other is equivalent to the effective motion with a single timelike dimension. The quantum constraints are consistent only in critical dimensions. For the bosonic system in a flat spacetime the critical dimension is 27 or 28 , with signature $(25,2)$ or $(26,2)$, depending on whether the particle is massive or massless, respectively. For the supersymmetric case the critical dimensions are 11 or 12 , with signature $(9,2)$ or $(10,2)$, under the same circumstances. Generalizations to multiparticles, strings, and $p$-branes are outlined. [S0556-2821(97)01318-0]
\end{abstract}

PACS number(s): 11.25.Hf, 04.20.Cv, 04.20.Jb

\section{CLASSICAL PARTICLES AND STRINGS}

The idea that the fundamental theory may be formulated in 12 or more dimensions has been receiving increased attention [1-19]. It has become apparent that some of the extra dimensions are timelike, and thus the issues associated with more than one timelike dimensions must be addressed seriously. As a first step toward theories with two or more timelike dimensions, in which the traditional problems are overcome, we have proposed a set of gauge symmetries and the associated constraints, as well as a cosmological scenario [13]. As an example, we formulated an action principle for two particles which move freely except for a global constraint on each other's momenta. In this paper we generalize this type of action principle by discussing the example of a string and a particle in detail, and then showing how to apply the same methods to more particles, strings, and $p$-branes. We discuss the quantum constraints, the emergence of critical dimensions, and the quantum consistent sectors. The supersymmetric generalization is outlined.

\section{A. Reformulation of the two particles}

Consider two particles described by their world lines $x_{1}^{\mu}(\tau), x_{2}^{\mu}(\tau)$. In our previous work [13], we presented an action with appropriate gauge invariances that produced the following constraints for the momenta of the two particles:

$$
p_{1}^{2}+m_{1}^{2}=0, p_{2}^{2}+m_{2}^{2}=0, p_{1} \cdot p_{2}=0 .
$$

The two particles move freely, except for the mutual constraint $p_{1} \cdot p_{2}=0$. Two orthogonal timelike momenta cannot exist in a space with a single timelike dimension. The extra constraint was the key for the two timelike dimensions and their interpretation given in Ref. [13]. In this section we would like to give another formulation of the action that

\footnotetext{
* On sabbatical leave from the Department of Physics and Astronomy, University of Southern California, Los Angeles, CA 90089-0484.

${ }^{\dagger}$ On leave from Ecole Normale Supérieure, 24 rue Lhomond, F-75231, Paris, Cedex 05, France.
}

leads to the same results. The new formulation is better adapted to generalizations to strings and $p$-branes.

We consider the following action for the two particles:

$$
\begin{gathered}
S=S_{1}\left(x_{1}, A_{1}, e_{1}, \lambda_{2}\right)+S_{2}\left(x_{2}, A_{2}, e_{2}, \lambda_{1}\right)+\lambda_{1}^{\mu} \lambda_{2}^{\nu} \eta_{\mu \nu}, \\
S_{1}=\frac{1}{2} \int_{0}^{T} d \tau\left[e_{1}^{-1}\left(\partial_{\tau} x_{1}^{\mu}-\lambda_{2}^{\mu} A_{1}\right)^{2}-e_{1} m_{1}^{2}\right],
\end{gathered}
$$

and similarly for $S_{2}$, where $x_{i}^{\mu}(\tau), A_{i}(\tau), e_{i}(\tau)$ are functions of $\tau$ while $\lambda_{i}^{\mu}$ are independent of $\tau$. Note that $\lambda_{2}^{\mu}$ appears in $S_{1}$ and $\lambda_{1}^{\mu}$ appears in $S_{2}$. As we will see, $\lambda_{2}^{\mu}$ is determined in terms of canonical variables that belong to particle No. 2 , so that the presence of particle No. 2 influences particle No. 1, and vice versa. In the path integral we integrate over the $\lambda_{i}^{\mu}$ as well as the other fields. Therefore, in the classical theory we minimize the action with respect to the $\lambda_{i}^{\mu}$ as well as the other fields.

The $\tau$ reparametrization invariance of $S_{i}\left(x_{i}, A_{i}, e_{i}, \lambda_{i^{\prime}}\right)$ is independent for each $i$ (we denote $1^{\prime}=2$ and $2^{\prime}=1$ ), hence there are two reparametrizations that eventually allow the two gauge choices $e_{i}(\tau)=1$. The equations of motion for the $e_{i}$ lead to two constraints

$$
p_{i}^{2}+m_{i}^{2}=0
$$

where

$$
p_{i}^{\mu}=e_{i}^{-1}\left(\partial_{\tau} x_{i}^{\mu}-\lambda_{i^{\prime}}^{\mu} A_{i}\right), \quad \partial_{\tau} p_{i}^{\mu}=0
$$

are the canonical momenta, which are conserved according to the equations of motion for $x_{i}^{\mu}$.

The action is gauge invariant under the following two gauge transformations with parameters $\Lambda_{i}(\tau)$ :

$$
\delta x_{i}^{\mu}(\tau)=\lambda_{i^{\prime}}^{\mu} \Lambda_{i}(\tau), \quad \delta A_{i}(\tau)=\partial_{\tau} \Lambda_{i}(\tau)
$$

The covariant derivatives $\partial_{\tau} x_{i}^{\mu}-\lambda_{i}^{\mu} A_{i}$ may be seen as arising from the gauging of an Abelian subgroup in the spirit of gauged Wess-Zumino-Witten (WZW) models. Because of these gauge invariances, the equations of motion for $A_{i}$ lead to two other constraints that help remove degrees of freedom 


$$
\lambda_{i^{\prime}} \cdot p_{i}=0 .
$$

Finally, we come to the role of the coupling term $\lambda_{1} \cdot \lambda_{2}$ in the action. If it were not for this coupling term there would be two independent Lorentz symmetries, one for each particle. However, because of this coupling there is a single Lorentz symmetry, in a $d$-dimensional space with a metric $\eta_{\mu \nu}$ whose signature will be determined by the solution of all the constraints. The equations of motion for the $\lambda_{i}$ give

$$
\lambda_{1}^{\mu}=\int_{0}^{T} d \tau A_{1} p_{1}^{\mu}, \quad \lambda_{2}^{\mu}=\int_{0}^{T} d \tau A_{2} p_{2}^{\mu} .
$$

Since the momenta are independent of $\tau$, one finds

$$
\lambda_{1}^{\mu} \sim p_{1}^{\mu}, \quad \lambda_{2}^{\mu} \sim p_{2}^{\mu} .
$$

Therefore, the two constraints in Eq. (6) reduce to the single constraint

$$
p_{1} \cdot p_{2}=0 .
$$

We have demonstrated that the new action in Eq. (2) reproduces the same system of constraints in Eq. (1) given by our old action. Hence, we need to introduce two timelike dimensions and interpret them as in our previous work. This reformulation is more elegant and permits generalizations to strings and $p$-branes, as discussed below.

\section{B. A string and a particle}

Consider a string and a particle described by a world sheet $x_{1}^{\mu}(\tau, \sigma)$ and a world line $x_{2}^{\mu}(\tau)$, respectively, and introduce the action

$$
\begin{aligned}
& S=S_{1}\left(x_{1}, A_{1 m}, g_{m n}, \lambda_{2}\right)+S_{2}\left(x_{2}, A_{2}, e_{2}, \lambda_{1}\right)+\lambda_{1} \cdot \lambda_{2}, \\
& S_{1}=\frac{1}{2} \int_{0}^{T} d \tau \int d \sigma \sqrt{-g} g^{m n}\left(\partial_{m} x_{1}^{\mu}-\lambda_{2}^{\mu} A_{1 m}\right) \\
& \times\left(\partial_{n} x_{1}^{\nu}-\lambda_{2}^{\nu} A_{1 n}\right) \eta_{\mu \nu}, \\
& S_{2}= \frac{1}{2} \int_{0}^{T} d \tau\left[e_{2}^{-1}\left(\partial_{\tau} x_{2}^{\mu}-\lambda_{1}^{\mu} A_{2}\right)^{2}-e_{2} m_{2}^{2}\right] .
\end{aligned}
$$

The two actions $S_{1,2}$ are invariant under independent reparametrizations, hence one can choose the usual conformal gauge for the string $\sqrt{-g} g^{m n}=\eta^{m n}$, and $e_{2}=1$ for the particle, and obtain the following constraints from the equations of motion of $g^{m n}, e_{2}$, respectively:

$$
\left(D_{ \pm} x_{1}^{\mu}\right)^{2}=0, p_{2}^{2}+m_{2}^{2}=0
$$

where

$$
\begin{gathered}
D_{ \pm} x_{1}^{\mu}=\left(\partial_{ \pm} x_{1}^{\mu}-\lambda_{2}^{\mu} A_{1 \pm}\right), \\
p_{2}^{\mu}=e_{2}^{-1}\left(\partial_{\tau} x_{2}^{\mu}-\lambda_{1}^{\mu} A_{2}\right),
\end{gathered}
$$

and the $\partial_{ \pm}$derivatives are with respect to the light cone variables $\sigma^{ \pm} \equiv \tau \pm \sigma$. The equations of motion for $x_{1}^{\mu}(\tau, \sigma), x_{2}^{\mu}(\tau)$, in the gauges we have chosen, are

$$
\partial_{+}\left(D_{-} x_{1}^{\mu}\right)+\partial_{-}\left(D_{+} x_{1}^{\mu}\right)=0, \quad \partial_{\tau} p_{2}^{\mu}=0 .
$$

As in the two particle case, there are additional gauge invariances, which may be understood in the spirit of gauged WZW models

$$
\begin{gathered}
\delta_{1} x_{1}^{\mu}=\lambda_{2}^{\mu} \Lambda_{1}(\tau, \sigma), \quad \delta_{1} A_{1 m}=\partial_{m} \Lambda_{1}(\tau, \sigma), \\
\delta_{2} x_{2}^{\mu}=\lambda_{1}^{\mu} \Lambda_{2}(\tau), \quad \delta_{2} A_{2}=\partial_{\tau} \Lambda_{2}(\tau),
\end{gathered}
$$

and these explain the structure of the covariant derivatives $\left(g_{m n}, e_{2}, \lambda_{1,2}^{\mu}\right.$ are invariant under $\left.\delta_{1,2}\right)$. The equations of motion for $A_{1 \pm}, A_{2}$ give the constraints associated with these gauge invariances

$$
\lambda_{2} \cdot D_{ \pm} x_{1}=0, \lambda_{1} \cdot p_{2}=0 .
$$

Finally, the equations of motion for $\lambda_{1,2}^{\mu}$ give

$$
\begin{gathered}
\lambda_{1}^{\mu}=\int_{0}^{T} d \tau \int d \sigma\left(D_{+} x_{1}^{\mu} A_{1-}+D_{-} x_{1}^{\mu} A_{1+}\right), \\
\lambda_{2}^{\mu}=\int_{0}^{T} d \tau A_{2} p_{2}^{\mu} .
\end{gathered}
$$

Using the constraints (15), we deduce

$$
\lambda_{1} \cdot \lambda_{2}=0 .
$$

Since $p_{2}^{\mu}$ is conserved, one finds $\lambda_{2}^{\mu} \sim p_{2}^{\mu}$.

From the first equation in Eq. (15) we deduce $A_{1 \pm}=\left(1 / \lambda_{2}^{2}\right) \lambda_{2} \cdot \partial_{ \pm} x_{1}$ which allows us to write

$$
D_{ \pm} x_{1}^{\mu}=\partial_{ \pm} x_{1}^{\mu}-\frac{1}{p_{2}^{2}} p_{2}^{\mu} p_{2} \cdot \partial_{ \pm} x_{1},
$$

which solves the constraint $\lambda_{2} \cdot D_{ \pm} x_{1}=p_{2} \cdot D_{ \pm} x_{1}=0$ explicitly, provided $p_{2}^{2} \neq 0$. From this form one sees that the component of $x_{1}^{\mu}$ that is parallel to $p_{2}^{\mu}$ drops out of the string system when $p_{2}^{2} \neq 0$. Using the $\Lambda_{1}(\tau, \sigma)$ gauge invariance one may choose the gauge

$$
p_{2} \cdot x_{1}(\tau, \sigma)=2 c_{1} p_{2}^{2} \tau=c_{1} p_{2}^{2}\left(\sigma^{+}+\sigma^{-}\right),
$$

where $c_{1}$ is a constant. In this gauge one has

$$
D_{ \pm} x_{1}^{\mu}=\partial_{ \pm} x_{1}^{\mu}-c_{1} p_{2}^{\mu}, \quad A_{1 \pm}(\tau, \sigma)=c_{1} \sqrt{p_{2}^{2} / \lambda_{2}^{2}},
$$

which leads to the simplification

$$
\lambda_{1}^{\mu}=c_{1} \sqrt{p_{2}^{2} / \lambda_{2}^{2}} \int_{0}^{T} d \tau \int d \sigma\left(\partial_{\tau} x_{1}^{\mu}-2 c_{1} p_{2}^{\mu}\right) .
$$

The canonical momentum density for the string is $D_{\tau} x_{1}^{\mu}=\partial_{\tau} x_{1}^{\mu}-A_{1 \tau} \lambda_{2}^{\mu}=\partial_{\tau} x_{1}^{\mu}-2 c_{1} p_{2}^{\mu}$. Inserting this in the equation above, one finds that $\lambda_{1}^{\mu}$ is proportional to the total conserved momentum of the string No. 1 ,

$$
p_{1}^{\mu}=\int d \sigma\left(\partial_{\tau} x_{1}^{\mu}-2 c_{1} p_{2}^{\mu}\right),
$$


and that it is orthogonal to the momentum of particle No. 2 . Since both $\lambda_{1}^{\mu}$ and $p_{1}^{\mu}$ are gauge-independent quantities, their relation, which was derived in a specific gauge, is also gauge invariant. Hence we have deduced that

$$
\lambda_{1}^{\mu} \sim p_{1}^{\mu}, \quad \lambda_{2}^{\mu} \sim p_{2}^{\mu}, \quad p_{1} \cdot p_{2}=0,
$$

just as in the case of two particles of the previous section.

In the lightlike case $\lambda_{2}^{2}=0=p_{2}^{2}$ (which is consistent only if $\left.m_{2}=0\right)$, the action $S_{1}$ has no $A_{1+} A_{1-}$ term, and acquires an additional gauge symmetry beyond Eq. (14):

$$
\delta_{3} x_{1}^{\mu}(\tau, \sigma)=0, \quad \delta_{3} A_{1 \pm}= \pm \partial_{ \pm} \Lambda_{3}(\tau, \sigma) .
$$

The constraint $p_{2} \cdot D_{ \pm} x_{1}=p_{2} \cdot \partial_{ \pm} x_{1}=0$ eliminates a component of $x_{1}^{\mu}(\tau, \sigma)$ not parallel to $p_{2}^{\mu}$ [this is consistent with gauge choice (19) although in this case it follows from the constraint]. The extra $\Lambda_{3}(\tau, \sigma)$ gauge symmetry can be used to gauge fix $A_{1 \pm}$ to $A_{1 \pm}=\partial_{ \pm} \gamma(\tau, \sigma)$ and then use gauge symmetry (14) to fix $\gamma(\tau, \sigma)$ so that $D_{ \pm} x_{1}$ and $A_{1 \pm}$ take the form in Eq. (20). In this way, all the results above, including Eq. (23), apply in the massless particle case as well, and this may be understood as the limit in which $p_{2}^{2} / \lambda_{2}^{2}$ remains finite. However, in the background of the massless particle, two string components, rather than only one, are eliminated by the gauge invariances: $\partial_{ \pm} x_{1}^{\mu}-c_{1} p_{2}^{\mu}$ has no components along the lightlike $p_{2}^{\mu}$, and $p_{2} \cdot \partial_{ \pm} x_{1}=0$, whereas for the massive particle these two conditions correspond to one and the same component. This kind of phenomenon happened also in the massless limit of the two particle case, as explained in Ref. [13].

The equation of motion for the string is easily solved since it has the free string form

$$
\partial_{+} \partial_{-} x_{1}^{\mu}=0
$$

As usual, the general solution is given in terms of left and right movers

$$
\begin{gathered}
x_{1 \mu}=x_{1 \mu}^{(+)}\left(\sigma^{+}\right)+x_{1 \mu}^{(-)}\left(\sigma^{-}\right)+c_{1} p_{2 \mu} \tau \\
x_{1 \mu}^{( \pm)}\left(\sigma^{ \pm}\right)=\frac{1}{2}\left(q_{1 \mu}+\frac{\sigma^{ \pm}}{2 \pi} p_{1 \mu}\right)-i \sum_{n \neq 0} \frac{1}{n} \alpha_{n \mu}^{( \pm)} e^{i n \sigma^{ \pm}} .
\end{gathered}
$$

The term proportional to $c_{1} p_{2}^{\mu} \tau$ is added so as to be consistent with the definition of the total string momentum $p_{1}^{\mu}$ that followed from the canonical formalism. The canonical pair is $\left(q_{1 \mu}, p_{1 \mu}\right)$, while the $\alpha_{n \mu}^{( \pm)}$have the usual string oscillator commutation rules.

The equations of motion for particle No. 2 are also solved easily since $p_{2}^{\mu}$ is conserved. The constraint in Eq. (15), together with the definition of $p_{2}^{\mu}$ in terms of the velocity $\partial_{\tau} x_{2}^{\mu}$, give $A_{2}(\tau)=\left(1 / \lambda_{1}^{2}\right) \lambda_{1} \cdot \partial_{\tau} x_{2}$, so that

$$
p_{2}^{\mu}=\partial_{\tau} x_{2}^{\mu}-p_{1}^{\mu} \frac{1}{p_{1}^{2}} p_{1} \cdot \partial_{\tau} x_{2}
$$

Using the $\Lambda_{2}(\tau)$ gauge invariance, one can choose the gauge

$$
p_{1} \cdot \partial_{\tau} x_{2}(\tau)=c_{2} p_{1}^{2}
$$

where $c_{2}$ is a constant. In this gauge the conserved momentum of particle No. 2 and $A_{2}$ become

$$
p_{2}^{\mu}=\partial_{\tau} x_{2}^{\mu}-c_{2} p_{1}^{\mu}, \quad A_{2}=c_{2} \sqrt{p_{1}^{2} / \lambda_{1}^{2}} .
$$

This form is valid for the massive as well as massless string states (i.e., $p_{1}^{2} / \lambda_{1}^{2}$ finite as $p_{1}^{2} \rightarrow 0$, as above). The solution of the particle equation is

$$
x_{2}^{\mu}(\tau)=\left(p_{2}^{\mu}+c_{2} p_{1}^{\mu}\right) \tau+q_{2}^{\mu},
$$

showing that it moves as a free particle, except for the orthogonality constraint $p_{1} \cdot p_{2}=0$. The canonical pair is $\left(q_{2}^{\mu}, p_{2}^{\mu}\right)$.

By reexamining the equations for $\lambda_{1,2}^{\mu}$ one finds that

$$
\lambda_{1}^{\mu}=T a_{1} p_{1}^{\mu}, \quad \lambda_{2}^{\mu}=T a_{2} p_{2}^{\mu},
$$

where $a_{1,2}$ are constant zero modes of the gauge fields that have survived in the general solution

$$
A_{1 \pm}(\tau, \sigma) \equiv a_{1}, A_{2}(\tau)=a_{2}
$$

and that the two constants $c_{1,2}$ are equal and given by $c_{1}=c_{2}=T a_{1} a_{2}$.

\section{QUANTIZATION AND CRITICAL DIMENSIONS}

In the previous section it was shown that the particle and string systems move as free systems except for a set of constraints. The canonical degrees of freedom $\left(q_{2}^{\mu}, p_{2}^{\mu}\right),\left(q_{1}^{\mu}, p_{1}^{\mu}\right), \alpha_{n \mu}^{ \pm}$satisfy the first class constraints that follow from Eqs. (11), (15), (23):

$$
\begin{gathered}
m_{2} \neq 0: \Phi \equiv p_{2}^{2}+m_{2}^{2}=0, L_{n}^{ \pm}=0, J_{0}^{ \pm} \equiv p_{2} \cdot p_{1}=0, \\
m_{2}=0: \Phi \equiv p_{2}^{2}=0, L_{n}^{ \pm}=0, J_{n}^{ \pm} \equiv p_{2} \cdot \alpha_{n}^{ \pm}=0,
\end{gathered}
$$

where the Virasoro operators are

$$
\begin{gathered}
m_{2} \neq 0: \quad L_{n}^{ \pm}=\frac{1}{2} \sum_{m=-\infty}^{\infty} \alpha_{n-m}^{( \pm) \mu} \cdot \alpha_{m}^{( \pm) \nu}\left(\eta_{\mu \nu}-\frac{p_{2 \mu} p_{2 \nu}}{p_{2}^{2}}\right), \\
m_{2}=0: \quad L_{n}^{ \pm}=\frac{1}{2} \sum_{m=-\infty}^{\infty} \alpha_{n-m}^{( \pm) \mu} \cdot \alpha_{m}^{( \pm) \nu} \eta_{\mu \nu} .
\end{gathered}
$$

We have used $\alpha_{0 \mu}^{ \pm}=p_{1 \mu}$, therefore, the constraint $J_{0}^{ \pm} \equiv p_{1} \cdot p_{2}=0$ is included above for $m_{2}=0$ as well. The second term in $L_{n}^{ \pm}$for $m_{2} \neq 0$ could be dropped since $p_{2} \cdot \alpha_{n}^{ \pm}=0$ was taken as a gauge choice, which may be added as an additional constraint $J_{n}^{ \pm} \equiv p_{2} \cdot \alpha_{n}^{ \pm}=0$ for the massive case as well. This may be done classically as well as in a light cone quantization, as we will see below. However, this gauge choice becomes a second class constraint in the massive case $m_{2} \neq 0$, that is harder to deal with in a covariant quantization (since it has an anomaly proportional to $m_{2}^{2}$ )

$$
\left[J_{n}^{ \pm}, J_{m}^{ \pm}\right]=n \Phi \delta_{n+m}-n m_{2}^{2} \delta_{n+m} .
$$

As we will see, this will play a role in the covariant quantization of the system and in the determination of the critical dimension. 
It is evident from the analogous two particle problem that there is no solution (neither classical nor quantum) unless there are two or more timelike dimensions. Assuming two timelike dimensions the signature of the space is given by $\eta_{\mu \nu}=\operatorname{diag}(-1,-1,1, \ldots, 1)$. Hence, there is a $\mathrm{SO}(d-2,2)$ covariance in $d$ dimensions. $d$ will be determined in the quantum theory. It will be fixed to a critical dimension $d_{\text {crit }}=27$ for $m_{2} \neq 0$, or $d_{\text {crit }}=28$ for $m_{2}=0$, to eliminate quantum anomalies.

We will first solve the constraints classically and hence we will add $J_{n}^{ \pm}=0$ as a gauge choice to the massive case as well. This solution will be used in a light cone quantization. Using the $\mathrm{SO}(d-2,2)$ symmetry one can boost $p_{2}^{\mu}$ to a timelike rest frame if the mass $m_{2} \neq 0$, or to a lightlike frame if the mass $m_{2}=0$,

$$
\hat{p}_{2}^{\mu}=\left(m_{2}, 0 ; \overrightarrow{0}, 0\right) \quad \text { or } \hat{p}_{2}^{\mu}=\left(\left|p_{2}\right|, 0 ; \overrightarrow{0}, p_{2}\right),
$$

where the first two entries are timelike and the rest are spacelike. This allows the solution of three constraints in the form

$$
\begin{array}{ll}
m_{2} \neq 0: & \hat{p}_{1 \mu}=\left(0, \hat{p}_{1 I}\right), \quad \hat{\alpha}_{\mu n}^{ \pm}=\left(0, \hat{\alpha}_{I n}^{ \pm}\right), \\
m_{2}=0: & \hat{p}_{1}^{\mu}=\left(0, \hat{p}_{1 I}, 0\right), \quad \hat{\alpha}_{\mu n}^{ \pm}=\left(0, \hat{\alpha}_{I n}^{ \pm}, 0\right),
\end{array}
$$

leaving one constraint to be solved

$$
L_{n}^{ \pm}=\frac{1}{2} \sum_{m=-\infty}^{\infty} \hat{\alpha}_{n-m}^{ \pm} \cdot \hat{\alpha}_{m}^{ \pm}=0,
$$

where $\hat{\alpha}_{I n}^{ \pm}$are the string variables in the rest frame or light cone frame of particle No. 2, and the index $I$ labels a subspace of signature $(-1,1, \ldots, 1)$ whose dimension is $(d-1)$ for $m_{2} \neq 0$ and $(d-2)$ for $m_{2}=0$. Therefore, there remains a Lorentz symmetry $\mathrm{SO}(d-2,1)$ for $m_{2} \neq 0$ and $\mathrm{SO}$ $(d-3,1)$ for $m_{2}=0$. Note that for $m_{2}=0$, the solutions $\hat{\alpha}_{\mu n}^{ \pm}, \hat{p}_{1}^{\mu}$ have no components along the lightlike $\hat{p}_{2}^{\mu}$. This is due to the gauge symmetry (24) as explained above.

\section{A. Covariant quantization}

To quantize covariantly and implement the constraints (32) on the states one may use the Becchi-Rouet-StoraTyutin (BRST) formalism. The BRST procedure is valid for the first class constraints. We will pretend as if it applies to both massless and massive cases with the constraints $\Phi$, $L_{n}^{ \pm}, J_{n}^{ \pm}$in order to illustrate the problem with the anomaly in Eq. (34), and will find out that this set of constraints works only for $m_{2}=0$. Then we will discuss separately the massive case by dealing directly only with the first class constraints (without the $J_{n}^{ \pm}$).

Corresponding to the constraints $\Phi, L_{n}^{ \pm}, J_{n}^{ \pm}$one introduces the ghosts $(B, C),\left(b_{n}^{ \pm}, c_{n}^{ \pm}\right)$, and $\left(\beta_{n}^{ \pm}, \gamma_{n}^{ \pm}\right)$. The algebra of the constraints and their anomalies are

$$
\begin{gathered}
{\left[L_{n}^{ \pm}, L_{m}^{ \pm}\right]=(n-m) L_{n+m}^{ \pm}+\frac{d}{12}\left(n^{3}-n\right) \delta_{n+m, 0},} \\
{\left[L_{n}^{ \pm}, J_{m}^{ \pm}\right]=-m J_{m}^{ \pm},}
\end{gathered}
$$

$$
\left[J_{n}^{ \pm}, J_{m}^{ \pm}\right]=n \Phi \delta_{n+m}-n m_{2}^{2} \delta_{n+m} .
$$

The BRST operator is

$$
\begin{aligned}
Q= & C \Phi+\sum_{ \pm} \sum_{n}\left[c_{-n}^{ \pm} L_{n}^{ \pm}+\gamma_{-n}^{ \pm} J_{n}^{ \pm}\right] \\
& -\frac{1}{2} \sum_{ \pm} \sum_{n, m}(n-m) c_{-n}^{ \pm} c_{-m}^{ \pm} b_{n+m}^{ \pm} \\
& -\frac{1}{2} \sum_{ \pm} \sum_{m} m \gamma_{-m}^{ \pm} \gamma_{m}^{ \pm} B \\
& -\sum_{ \pm} \sum_{n, m}(-m) c_{-n}^{ \pm} \gamma_{-m}^{ \pm} \beta_{n+m}^{ \pm}-\sum_{ \pm} c_{0}^{ \pm} a^{ \pm}-C A,
\end{aligned}
$$

where $a^{ \pm}, A$ are anomaly constants to be determined by requiring $Q^{2}=0$. By anticommuting $Q$ with the antighosts $B, b_{n}, \beta_{n}$, one gets the total gauge generators of the BRSTquantized theory

$$
\begin{gathered}
\Phi^{\mathrm{tot}}=\Phi-A, \\
L_{n}^{( \pm) \mathrm{tot}=} L_{n}^{ \pm}+\sum_{m}(n-m) b_{n+m}^{ \pm} c_{-m}^{ \pm}+\sum_{m}(-m) \beta_{n+m}^{ \pm} \gamma_{-m}^{ \pm} \\
-a^{ \pm} \delta_{n 0}, \\
J_{n}^{( \pm) \text {tot }}=J_{n}^{ \pm}+n\left(\gamma_{n}^{ \pm} B+\sum_{m} \beta_{n+m}^{ \pm} c_{-m}^{ \pm}\right) .
\end{gathered}
$$

Requiring $Q^{2}=0$ is equivalent to requiring the total generators to close without anomalies. For a ghost system of dimension $h$ the Virasoro generator is $L_{n}^{(h)}$ $=\Sigma_{m}[n(h-1)-m] b_{n+m}^{(h)} c_{-m}^{(1-h)}$ and the anomaly in its algebra is

$$
\begin{gathered}
\operatorname{anom}(h)=\delta_{n+m} \frac{1}{12}\left[n^{3} c_{h}+2 n\right], \\
c_{h}=-12 h^{2}+12 h-2 .
\end{gathered}
$$

Therefore, the total anomaly in the algebra of $L_{n}^{( \pm) \text {tot }}$ is

$$
\frac{n^{3}}{12}\left(d+c_{h=2}+c_{h=1}\right)+\frac{n}{12}\left(-d+24 a^{ \pm}+2+2\right),
$$

with the total central charge

$$
c_{\text {tot }}=d+c_{h=2}+c_{h=1}=d-28 .
$$

The $J_{n}^{( \pm) \text {tot }}$ algebra is

$$
\left[J_{n}^{( \pm) \text {tot }}, J_{m}^{( \pm) \text {tot }}\right]=n \Phi^{\text {tot }} \delta_{n+m}+n\left(A-m_{2}^{2}\right) \delta_{n+m},
$$

and

$$
\left[L_{n}^{( \pm) \text {tot }}, J_{m}^{( \pm) \text {tot }}\right]=-m J_{n+m}^{( \pm) \text {tot }} .
$$

So, the total anomaly cancels if

$$
d=28, a^{ \pm}=1, A=m_{2}^{2} .
$$


Under these conditions one finds

$$
\Phi^{\mathrm{tot}}=p_{2}^{2}+m_{2}^{2}-A=p_{2}^{2}=0 .
$$

So, the quantum particle must be massless $p_{2}^{2}=0$, and the critical dimension for the total system is 28 . The global Lorentz symmetry of this quantum system is $\mathrm{SO}(26,2)$. Therefore, the analysis applies correctly only to the $m_{2}=0$ case, as anticipated above.

For $m_{2} \neq 0$, the correct treatment of the second class constraint $J_{n}^{( \pm)}=0$ needs more care. Since the present model is in the class of gauged WZW models, one may imitate the BRST procedure advocated in Ref. [20] to show that the final result is equivalent to the standard coset construction. In the coset language our case corresponds to $G / H$ with $G=R^{d}$ and $H=R$, and our Virasoro generator in Eq. (33) is indeed the coset construction for the conformal field theory for $R^{d} / R$. The central charge of this Virasoro generator is $d-1$, and for the quantum consistency of the conformal field theory it must be set equal to 26. Hence,

$$
m_{2} \neq 0: \quad d_{\text {crit }}=27,
$$

and the global symmetry of the system with a massive particle No. 2 is $\operatorname{SO}(25,2)$.

This result may be obtained more directly without appealing to the formalism of Ref. [20]. Namely, one can avoid the $J_{n}^{( \pm)}=0$ gauge fixing and work directly with the oscillators

$$
\widetilde{\alpha}_{n \mu}^{ \pm \pm}=\alpha_{n \mu}^{( \pm)}-\frac{1}{p_{2}^{2}} p_{2 \mu} p_{2} \cdot \alpha_{n}^{( \pm)}
$$

since these $\widetilde{\alpha}_{n \mu}^{( \pm)}$solve explicitly the original constraints $p_{2} \cdot D_{ \pm} x_{1}=0$. The Virasoro constraints in Eq. (33) are written directly in terms of these oscillators

$$
m_{2}=0: \quad L_{n}^{ \pm}=\frac{1}{2} \sum_{m=-\infty}^{\infty} \widetilde{\alpha}_{n-m}^{( \pm) \mu} \cdot \widetilde{\alpha}_{m}^{( \pm) \nu} \eta_{\mu \nu},
$$

and their commutation rules are

$$
\left[\widetilde{\alpha}_{n}^{( \pm) \nu}, \widetilde{\alpha}_{m}^{( \pm) \nu}\right]=n \delta_{n+m}\left(\eta^{\mu \nu}-\frac{p_{2}^{\mu} p_{2}^{\nu}}{p_{2}^{2}}\right) .
$$

The only constraints that need to be considered are the first class constraints $L_{n}^{ \pm}=0, \Phi=p_{2}^{2}+m_{2}^{2}=0, J_{0}=p_{1} \cdot p_{2}=0$. In the algebra of these constraints all commutators are zero, except for

$$
\left[L_{n}^{ \pm}, L_{m}^{ \pm}\right]=(n-m) L_{n+m}^{ \pm}+\frac{d-1}{12}\left(n^{3}-n\right) \delta_{n+m, 0},
$$

where the $d-1$ anomaly comes from

$$
\left(\eta^{\mu \nu}-\frac{p_{2}^{\mu} p_{2}^{\nu}}{p_{2}^{2}}\right)\left(\eta_{\mu \nu}-\frac{p_{2 \mu} p_{2 \nu}}{p_{2}^{2}}\right)=d-1 .
$$

The BRST operator is then

$$
\begin{aligned}
Q= & \sum_{ \pm} \sum_{n} c_{-n}^{ \pm} L_{n}^{ \pm}-\frac{1}{2} \sum_{ \pm} \sum_{n, m}(n-m) c_{-n}^{ \pm} c_{-m}^{ \pm} b_{n+m}^{ \pm}+C \Phi \\
& +\gamma_{0} J_{0}-\sum_{ \pm} c_{0}^{ \pm} a^{ \pm}
\end{aligned}
$$

and the standard procedure gives the critical dimension $d-1=26$.

\section{B. Light cone quantization}

The remaining constraints in the rest frame or light cone frame of particle No. 2 are $\left(\partial_{ \pm} \hat{x}_{1 \pm}^{I}\right)^{2}=0$ or $L_{n}^{ \pm}=0$ of Eq. (37). These are the familiar Virasoro constraints of string theory, which can be solved explicitly by taking advantage of the conformal invariance of the string system and choosing the light cone gauge $\hat{x}_{1}^{+}=\hat{p}_{1}^{+} \tau$,

$$
\begin{gathered}
\hat{\alpha}_{n}^{( \pm)-}=\frac{1}{2 \hat{p}_{1}^{+}} \sum_{m=-\infty}^{\infty} \sum_{I} \hat{\alpha}_{n-m}^{( \pm) i} \hat{\alpha}_{m}^{( \pm) i}-\frac{\widetilde{a}}{\hat{p}_{1}^{+}} \delta_{n, 0}, \\
\hat{\alpha}_{n}^{( \pm)+}=\hat{p}_{1}^{+} \delta_{n, 0} .
\end{gathered}
$$

The unconstrained degrees of freedom are the transverse string oscillators $\vec{\alpha}_{n}^{( \pm) i}$ that describe the left or right moving string excitations and the center-of-mass canonical degrees of freedom for the string $\left(\hat{q}_{1}, \hat{p}_{1}\right)$ and particle $\left(\hat{q}_{2}, \hat{p}_{2}\right)$, all of which are expressed in the rest frame or light cone frame of particle No. 2.

The Lorentz symmetry $\mathrm{SO}(d-2,1)$ [or $\mathrm{SO}(d-3,1)]$, which was manifest in the special frame [Eqs. (35)-(37)], is hidden in the light cone gauge (55) for the string. As is well known, the quantum algebra for the normal-ordered generators $\hat{M}^{I J}=\left(\hat{M}^{-+}, \hat{M}^{i+}, \hat{M}^{i-}, \hat{M}^{i j}\right)$,

$$
\begin{gathered}
\hat{M}^{-+}=\hat{q}_{1}^{-} \hat{p}_{1}^{+}, \quad \hat{M}^{i+}=\hat{q}_{1}^{i} \hat{p}_{1}^{+}, \\
\hat{M}^{i-}=\hat{q}_{1}^{i} \hat{p}_{1}^{-}-\hat{q}_{1}^{-} \hat{p}_{1}^{i}-i \sum_{ \pm} \sum_{n \neq 0} \frac{1}{n}: \hat{\alpha}_{-n}^{( \pm) i} \hat{\alpha}_{n}^{( \pm)-}:,
\end{gathered}
$$

$$
\hat{M}^{i j}=\hat{q}_{1}^{i} \hat{p}_{1}^{j}-\hat{q}_{1}^{j} \hat{p}_{1}^{i}-i \sum_{ \pm} \sum_{n \neq 0} \frac{1}{n}: \hat{\alpha}_{-n}^{( \pm) i} \hat{\alpha}_{n}^{( \pm) j}:
$$

of this symmetry closes correctly only if the number of transverse dimensions labeled by $i$ is 24 and $\tilde{a}=1$ (see, e.g., Ref. [21]). The number of transverse dimensions is $(d-3)=24$ for $m_{2} \neq 0$, and $(d-4)=24$ for $m_{2}=0$. Therefore, the particle and string system has a critical dimension

$$
\begin{aligned}
& m_{2} \neq 0: \quad d_{\text {crit }}=27, \\
& m_{2}=0: \quad d_{\text {crit }}=28,
\end{aligned}
$$

so the generators $\hat{M}^{I J}$ represent correctly $\mathrm{SO}(25,1)$ at the quantum level.

The original action was invariant under the full classical rotation invariance $\mathrm{SO}(d-2,2)$. For the critical dimension this classical symmetry is $\operatorname{SO}(25,2)$ if $m_{2} \neq 0$ and $\mathrm{SO}(26,2)$ if $m_{2}=0$. We now need to show that the quantum 
theory has the higher symmetry by verifying that the Lorentz algebra closes. To construct the remaining generators of $\mathrm{SO}(25,2)$ [or $\mathrm{SO}(26,2)]$, we need to boost back to the general frame of particle No. 2 and include the canonical degrees of freedom of particle No. 2.

For the massive particle $m_{2} \neq 0$ in the general frame, the mass shell constraint is solved by the $\mathrm{SO}(d-2,2)$ covariant vector

$$
p_{2}^{\mu}=\left(E_{2}^{\prime}, p_{2}^{I}\right), \quad E_{2}^{\prime}=\sqrt{p_{2} \cdot p_{2}+m_{2}^{2}},
$$

where $p_{2}^{I}$ is a $\mathrm{SO}(25,1)$ vector. The boost of any vector $\hat{v}^{\mu}=\left(\hat{v}^{0^{\prime}}, \hat{v}^{I}\right)$ defined in the particle rest frame (denoted with the hats) to the vector $v^{\mu}=\left(v^{0^{\prime}}, v^{I}\right)$ defined in the particle general frame (no hat) is given by

$$
\begin{gathered}
v^{0^{\prime}}=\frac{1}{m_{2}}\left(E_{2}^{\prime} \hat{v}^{0^{\prime}}+p_{2} \cdot \hat{v}\right), \\
v^{I}=\hat{v}^{I}+\frac{p_{2}^{I}}{m_{2}}\left(\frac{p_{2} \cdot \hat{v}}{E_{2}^{\prime}+m_{2}}+\hat{v}^{0^{\prime}}\right),
\end{gathered}
$$

where the sum over $I$ in the dot products is $\mathrm{SO}(25,1)$ covariant in 26 dimensions. Of course, the transformation is such that dot products are $\mathrm{SO}(25,2)$ invariant in the full 27 dimensions $\hat{v}^{2}=v^{2}$. The string and particle can now be described in the general frame by boosting the rest frame solution in Eqs. (35)-(37). Taking into account $\hat{\alpha}_{n}^{( \pm) 0^{\prime}}=0$, one obtains

$$
\alpha_{n}^{( \pm) 0^{\prime}}=\frac{p_{2} \cdot \hat{\alpha}_{n}^{ \pm}}{m_{2}}, \quad \alpha_{n}^{( \pm) I}=\hat{\alpha}_{n}^{( \pm) I}+\frac{p_{2}^{I}}{m_{2}} \frac{p_{2} \cdot \hat{\alpha}_{n}^{ \pm}}{E_{2}^{\prime}+m_{2}} .
$$

These are expected to form covariant $\mathrm{SO}(25,2)$ vectors $\alpha_{n}^{( \pm) \mu}=\left(\alpha_{n}^{( \pm) 0^{\prime}}, \alpha_{n}^{( \pm) I}\right)$, as will be verified below. Furthermore, because of the $\mathrm{SO}(25,2)$ invariance of dot products, the fully $\mathrm{SO}(25,2)$ covariant Virasoro constraints in Eq. (33) are equal to Eq. (37) for any $p_{2}^{\mu}=\left(E_{2}^{\prime}, p_{2}^{I}\right)$. Therefore, the explicit solution of these constraints is given in terms of only the 24 transverse oscillators in Eq. (55). Thus, the $27 \mathrm{com}$ ponents $\alpha_{n}^{( \pm) \mu}$ given in Eq. (60) are also expressed in terms of the 24 oscillators in Eq. (55) which are the ones that solve all the constraints in the general frame of the massive particle. So, for example, the $0^{\prime}$ component is

$$
\alpha_{n}^{( \pm) 0^{\prime}}=\frac{1}{m_{2}}\left(-p_{2}^{+} \cdot \hat{\alpha}_{n}^{( \pm)-}-p_{2}^{-} p_{1}^{+} \delta_{n, 0}+p_{2}^{i} \alpha_{n}^{( \pm) i}\right),
$$

where $\hat{\alpha}_{n}^{( \pm)-}$is quadratic in the 24 transverse oscillators as given in Eq. (55).

We are now ready to construct the generators $M^{\mu \nu}=\left(M^{0^{\prime} I}, M^{I J}\right)$ of $\mathrm{SO}(25,2)$ in the general frame of the massive particle No. 2 . They are given by

$$
M^{0^{\prime} I}=\frac{1}{2}\left(q_{2}^{I} E_{2}^{\prime}+E_{2}^{\prime} q_{2}^{I}\right)+\frac{p_{2 J} \hat{M}^{J I}}{E_{2}^{\prime}+m_{2}},
$$

$$
M^{I J}=q_{2}^{I} p_{2}^{J}-q_{2}^{J} p_{2}^{I}+\hat{M}^{I J},
$$

where $\hat{M}^{I J}$, which satisfy the $\operatorname{SO}(25,1)$ Lie algebra, are given in terms of the 24 transverse string oscillators in Eq. (56). It can be checked that these $M^{\mu \nu}$ satisfy the $\operatorname{SO}(25,2)$ Lie algebra without any anomalies, and furthermore, that they rotate the $\alpha_{n}^{( \pm) \mu}$ of Eq. (60) as vectors

$$
\left[M^{\mu \nu}, \alpha_{n}^{( \pm) \lambda}\right]=i \eta^{\mu \lambda} \alpha_{n}^{( \pm) \nu}-i \eta^{\nu \lambda} \alpha_{n}^{( \pm) \mu} .
$$

This last property is trivial for the $M^{I J}, \alpha_{n}^{( \pm) K}$ since it is the same as the usual 26-dimensional string in the light cone gauge. The new feature is the structure of $M^{0^{\prime} I}$. It can be checked that this structure automatically closes into the higher algebra $\mathrm{SO}(25,2)$ provided the $\hat{M}^{I J}$ form the $\mathrm{SO}(25,1)$ Lie algebra.

The form of $M^{0^{\prime} I}$ follows from rather general properties of cosets. The $\alpha_{n}^{( \pm) \mu}$ are given by boosting the $\hat{\alpha}_{n}^{( \pm) \mu}$ with a $p_{2}^{\mu}$-dependent boost

$$
\alpha_{n}^{( \pm) \mu}=T_{\nu}^{\mu}\left(p_{2}\right) \hat{\alpha}_{n}^{( \pm) \nu},
$$

where $T_{\nu}^{\mu}\left(p_{2}\right)$ is in the coset $\operatorname{SO}(25,2) / \operatorname{SO}(25,1)$. When a general $\mathrm{SO}(25,2)$ transformation is applied, it can be rewritten as

$$
\alpha_{n}^{( \pm) \mu} \rightarrow \Lambda_{\nu}^{\mu} \alpha_{n}^{( \pm) \nu}=\left[\Lambda T\left(p_{2}\right)\right]_{\nu}^{\mu} \hat{\alpha}_{n}^{( \pm) \nu}=\left[T\left(p_{2}^{\prime}\right) H\right]_{\nu}^{\mu} \hat{\alpha}_{n}^{( \pm) \nu},
$$

where $p_{2}^{\prime \mu}=\Lambda_{\nu}^{\mu} p_{2}^{\nu}$, and $H\left(p_{2}, \Lambda\right)$ is an element in the subgroup $\mathrm{SO}(25,1)$ but its parameters depend on a function of both $p_{2}^{\mu}$ and $\Lambda_{\nu}^{\mu}$. When $\Lambda$ is an element of $\operatorname{SO}(25,1)$, one has $H=\Lambda$, therefore, the subgroup is implemented on the 26 $\hat{\alpha}_{n}^{( \pm) I}$ and $p_{2}^{I}$ by the total particle and string generators $M^{I J}$. Of course, the $\hat{\alpha}_{n}^{( \pm) 0^{\prime}}$ is invariant under this combined transformation since it is a dot product. The remaining $\mathrm{SO}(25,2) / \mathrm{SO}(25,1)$ coset transformations have generators that are precisely the $M^{0^{\prime} I}$ given above, and they automatically take into account the complicated nature of $H\left(p_{2}, \Lambda\right)$.

The outcome of the light cone quantization for $m_{2} \neq 0$ is a critical dimension $d=27$ with signature $(25,2)$, in agreement with the covariant quantization.

The light cone quantization for the $m_{2}=0$ case can be done in a similar way. One needs to boost back from the light cone frame of particle No. 2 to the general frame. It is a straightforward exercise and there are no oscillator ordering problems, just as in the massive case. Therefore, the critical dimension is $d=28$ with signature $(26,2)$, in agreement with the BRST quantization.

\section{MULTIPARTICLES, STRINGS, $p$-BRANES}

The type of action discussed in this paper can be generalized to other systems. For example, for three particles

$$
S=S_{1}+S_{2}+S_{3}+\lambda_{1} \cdot \lambda_{2}+\lambda_{2} \cdot \lambda_{3}+\lambda_{3} \cdot \lambda_{1},
$$

where $S_{1}\left(x_{1}^{\mu}, A_{12}, A_{13}, e_{1}, \lambda_{2}^{\mu}, \lambda_{3}^{\mu}\right)$ is the action for particle No. 1 in the background of particles Nos. 2,3, constructed in terms of gauge-covariant derivatives 


$$
D_{\tau} x_{1}^{\mu}=\partial_{\tau} x_{1}^{\mu}-A_{12} \lambda_{2}^{\mu}-A_{13} \lambda_{3}^{\mu}
$$

in the spirit of gauged WZW models. So, the coset is $R^{d} / R^{2}$. Similarly for the actions $S_{2,3}$ which are obtained by a cyclic permutation of the indices $1,2,3$. Because of the gauge invariances one finds constraints, and going through a similar analysis as the two particle case, one determines $\lambda_{i}^{\mu} \sim p_{i}^{\mu}$ and the constraints

$$
p_{i} \cdot p_{j}+m_{i}^{2} \delta_{i j}=0
$$

The solution of this system of constraints requires three timelike coordinates. In the bosonic case there seems to be no limit on the number of particles and corresponding new timelike dimensions, but with supersymmetry there are hints for both sufficient and necessary reasons to have a minimum as well as a maximum of three timelike dimensions in a setting that is $\operatorname{SO}(11,3)$ covariant [14]. The structures of Ref. [14] were found to be necessary and sufficient to unify type-A and type-B supersymmetries. This unification is possible with a minimum of three timelike dimensions and extending the general structure beyond 14 dimensions is not required by any known phenomena. Furthermore, there seems to be an obstruction to Yang-Mills supersymmetric systems beyond 14 dimensions [16], thus providing a hint for a maximum of three timelike dimensions.

The same approach can be applied to a string and the two particles, with results that can be guessed from the previously discussed cases, of a string and one particle, and the three particles. The general result is that when a massive particle is added one needs to add $(0,1)$ dimensions, i.e., one timelike dimension but no spacelike dimensions, and when a massless particle is added one must add $(1,1)$, i.e., one time plus one space dimensions. Similarly, one may substitute a membrane for a string, and so on for other $p$-branes, in the case of classical $p$-branes. Of course, one does not know how to solve the quantum theory that includes membranes or $p$-branes and, therefore, there is no reliable statement on the number of dimensions for which the quantum theory is consistent. However, there are partial hints that $d_{\text {crit }}=11$ for supermembranes [22], therefore, for the combined supermembrane and superparticle system, one may extrapolate these hints to $d=12$ with signature $(10,2)$ if the superparticle is massive or $d=13$ with signature $(11,2)$ if the superparticle is massless. The supersymmetry of such a system is not standard, as discussed in Ref. [23] and the next section.

Next, we consider two strings, with an action $S=S_{1}+S_{2}+\lambda_{1} \cdot \lambda_{2}$, where both $S_{1,2}$ are string actions of the form (10). The equations of motion and constraints for each string can be solved, both classically and quantum mechanically, following the same steps as Sec. I B and Sec. II. But now we find new features in the quantum consistency of the combined system. Recall that in consistent sectors the masses of each string are given by $-p_{i}^{2}=N_{i}-1$, where $N_{i}$ are the oscillator excitation levels. Perhaps the simplest way to arrive at the critical dimension is to note that a quantum consistent string must have 26 dimensions after putting the other string in one of its massive or massless states. If the second string is in a massless state, its effect on the first string is the same as a massless particle. Then the total number of dimensions for string No. 1 has to be 28 with signature
$(26,2)$, as we have shown in Sec. II. On the other hand, if the second string is in a massive state, its effect on the first string is the same as a massive particle, and the number of dimensions must be 27 , with signature $(25,2)$. There is also a third case when the mass of the second string is tachyonic. Then the number of dimensions is also 27 , but with signature $(26,1)$. The roles of the two strings may be reversed and similar statements would be made for the critical dimension of string No. 2. These statements cannot be all simultaneously right in the same theory, since the classical theory is defined with a fixed number of dimensions for both strings. Hence, as the consistent quantum sectors, we must select only the mass sectors that are simultaneously consistent for both strings for a fixed number of dimensions. The sectors are defined by whether the masses $\left(-p_{1}^{2},-p_{2}^{2}\right)$ are simultaneously zero, positive, or negative, and evidently the only consistent sectors are

$$
\begin{gathered}
(26,2): \quad\left(-p_{1}^{2},-p_{2}^{2}\right)=(0,0), \\
(25,2): \quad\left(-p_{1}^{2},-p_{2}^{2}\right)=(+,+), \\
(26,1): \quad\left(-p_{1}^{2},-p_{2}^{2}\right)=(-,-) .
\end{gathered}
$$

The $(0,0)$ sector which is possible in 28 dimensions has only one state, similarly the $(-,-)$ sector in 27 dimensions has only one state, while the $(+,+)$ sector in 27 dimensions has an infinite number of massive states from each string. Here we have assumed that the conformal field theory for each string has no spectator sectors, that is that all degrees of freedom of both strings are coupled via the coupling $\lambda_{1} \cdot \lambda_{2}$. Of course, this assumption can be modified by changing the model.

Consider a model that has one dimension for string No. 1 which remains uncoupled, while all other $\left(d_{1}-1\right)$ dimensions of string No. 1 are coupled to the $d_{2}$ dimensions of string No. 2 via $\lambda_{1} \cdot \lambda_{2} \sim p_{1} \cdot p_{2}$, so that $d_{2}=\left(d_{1}-1\right)$. So, string No. 1 has one extra dimension. We need to consider again the values of $\left(-p_{1}^{2},-p_{2}^{2}\right)$. The mass shell conditions in consistent sectors are now $-p_{1}^{2}+\tilde{p}^{2}=N_{1}-1$ and $-p_{2}^{2}=N_{2}-1$, where $\tilde{p}^{2}$ is the zero mode of the extra dimension of string No. 1 . If this dimension is compact there would be contributions from the winding sectors as well. In this model the sector $\left(-p_{1}^{2},-p_{2}^{2}\right)=(+, 0)$ is consistent for $d_{1}=28$ with signature $(26,2)$ and $d_{2}=27$ with signature $(25,2)$. There are an infinite number states from string No. 1 and only one state from string No. 2. Evidently, one can construct various consistent models provided the sectors are selected as above.

However, it is not clear that such sectors are selfconsistent by themselves under interactions. It is not yet clear what interactions should be considered. If all interactions defined through vertex operator products of both strings are included, then the sectors identified above do not seem to remain isolated from others. Perhaps one can make sense of interactions that mix sectors of different dimensions and signatures. More study is required to understand such issues. These questions did not arise for the string and particle or string and the two particle systems. 


\section{SUPERSYMMETRY}

In a separate publication the supersymmetric version of the superstring and a massive or massless superparticle will be discussed in detail [23]. This involves a construction of an action for the massive superparticle and a more general superstring action that is invariant under a generalized supersymmetry. Here we wish to mention some generalities and ideas for future applications and improvements. By extrapolating from the results of the present paper to the supersymmetric case, one expects critical dimensions $d=12$ for $m_{2}=0$ and $d=11$ for $m_{2} \neq 0$ with a Lorentz symmetry $\mathrm{SO}(10,2)$ and $\mathrm{SO}(9,2)$, respectively. Furthermore, as expected, quite generally from Ref. [5], and from discussions in Refs. $[12,13,16]$, the generalized superalgebra has to be

$$
\left\{Q_{\alpha}, Q_{\beta}\right\}=\gamma_{\alpha \beta}^{\mu \nu} p_{1 \mu} p_{2 \nu},
$$

where $Q_{\alpha}$ is the Majorana-Weyl spinor of $\mathrm{SO}(10,2)$ or $\operatorname{SO}(9,2)$ with 32 real components. In the light cone frame of the massless particle No. 2 (as well as in the rest frame of the massive particle No. 2), the remaining Lorentz symmetry is $\mathrm{SO}(9,1)$ and the supersymmetry reduces to the standard form of the ten-dimensional type IIA. Furthermore, the superstring reduces to the usual ten-dimensional type IIA string. By contrast, in the general frame, or in the action, there is full covariance under $\mathrm{SO}(10,2)$ or $\mathrm{SO}(9,2)$.

We believe that the massless system $m_{2}=0$, underlies a supergravity theory in 12 dimensions, with bilocal fields $\Phi\left(x_{1}, x_{2}\right)$ describing the string-particle system, along the lines first suggested in Ref. [5]. This supergravity theory has been partially realized in the special light cone frame in Ref. [17], but in a single Kaluza-Klein mode of the bilocal fields
$\Phi\left(x_{1}, x_{2}\right)$ (that is particle No. 2 has been frozen to be at a fixed momentum $p_{2}^{\mu}$ ). Similar considerations for the two particles (rather than string and particle) underlie the YangMills theories in 12 dimensions, which have also been only partially realized in a similar Kaluza-Klein mode [7].

As has been argued in Ref. [14], the unification of type-A and type-B supersymmetries point to a unifying supersymmetric structure in 14 dimensions with signature $(11,3)$. Some sectors of such a structure can be constructed by considering three superparticles, or a superstring with two superparticles, etc. It is expected from Ref. [14], and it has been confirmed in Ref. [16], that the three superparticle system underlies a super Yang-Mills theory in 14 dimensions. This theory has been partially constructed [15] in a Kaluza-Klein sector in the same sense as the two particle case (i.e., the sector in which the momenta of the two particles out of three are frozen). A superstring and two superparticles probably underlie a supergravity in 14 dimensions that would generalize [17] to 14 dimensions. A more general approach that includes all Kaluza-Klein modes has been illustrated in Ref. [12] for free fields. This approach needs to be further developed to include interactions by figuring out the calculus of representations of the new supersymmetry (70). It could then be applied to the construction of the full 12- or 14dimensional supergravity and super Yang-Mills theories in all Kaluza-Klein sectors.

\section{ACKNOWLEDGMENTS}

The research of I.B. was partially supported by a U.S. DOE Grant No. DE-FG03-84ER40168, and by USC, while that of C.K. was partially supported by EEC Grant No. ERBFMRX-CT 96/0045.
[1] M. Duff and M. P. Blencowe, Nucl. Phys. B310, 387 (1988).

[2] I. Bars, Phys. Rev. D 54, 5203 (1996); I. Bars, "Duality and hidden dimensions," in the Proceedings of the Conference Frontiers in Quantum Field Theory, Toyonaka, Japan, 1995 (unpublished), hep-th/9604200.

[3] C. Vafa, Nucl. Phys. B469, 403 (1996); D. R. Morrison and C. Vafa, ibid. B473, 74 (1996); E. Witten, ibid. B471, 195 (1996); A. Kumar and C. Vafa, Phys. Lett. B 396, 85 (1997).

[4] D. Kutasov and E. Martinec, Nucl. Phys. B477, 652 (1996); B477, 675 (1996); hep-th/9612102.

[5] I. Bars, Phys. Rev. D 55, 2373 (1997); I. Bars, in Proceedings of Second Sakharov Conference 1996, and Strings-96 Conference (unpublished), hep-th/9608061.

[6] E. Martinec, "Geometrical structures of M-theory,' hep-th/9608017.

[7] H. Nishino and E. Sezgin, Phys. Lett. B 388, 569 (1996).

[8] V. Periwal, Phys. Rev. D 55, 1711 (1997).

[9] S. Hewson and M. Perry, Nucl. Phys. B492, 249 (1997).

[10] A. A. Tseytlin, Phys. Rev. Lett. 78, 1864 (1997).

[11] I. Bars, Phys. Rev. D 55, 3633 (1997).

[12] I. Bars and C. Kounnas, "A new supersymmetry,", hep-th/9612119.
[13] I. Bars and C. Kounnas, Phys. Lett. B 402, 25 (1997).

[14] I. Bars, “A case for 14 dimensions," hep-th/9704054.

[15] I. Rudychev and E. Sezgin, "Superparticles in $D>11$," hep-th/9704057.

[16] E. Sezgin, "Super Yang-Mills in $(11,3)$ dimensions," hep-th/9703123.

[17] H. Nishino, "Supergravity in $10+2$ dimensions ...,", hep-th/9703214.

[18] J. de Boer and K. Skenderis, "Self-Dual Supergravity from $N=2$ Strings,' hep-th/9704040.

[19] P. K. Townsend, "Membrane tension and manifest IIB S-duality," hep-th/9705160.

[20] D. Karabali and H. Schnitzer, Nucl. Phys. B329, 649 (1990).

[21] M. B. Green, J. H. Schwarz, and E. Witten, Superstring Theory (Cambridge University Press, Cambridge, England, 1987), Vol. I.

[22] I. Bars, C. N. Pope, and E. Sezgin, Phys. Lett. B 198, 455 (1987); I. Bars, Nucl. Phys. B308, 462 (1988); I. Bars and C. N. Pope, Class. Quantum Grav. 5, 1157 (1988); Gen. Relativ. Gravit. 21, 545 (1989).

[23] I. Bars and C. Daliduman, "Superstrings with new supersymmetry in $(9,2)$ and $(10,2)$ dimensions,' hep-th/9707215. 